\title{
Der Chief Digital Officer (CDO): Eine empirische Untersuchung
}

\author{
Manuela Walchshofer · René Riedl
}

Eingegangen: 4. September 2016 / Angenommen: 18. Februar 2017 / Online publiziert: 20. April 2017 (C) Der/die Autor(en) 2017. Dieser Artikel ist eine Open-Access-Publikation.

Zusammenfassung Der Chief Digital Officer (CDO), eine neuartige ManagementPosition, wird zunehmend geschaffen, um die digitale Transformation in Unternehmen zu planen und umzusetzen. Angesichts dieser Entwicklung stellt sich die Frage, ob nicht auch der Chief Information Officer (CIO) die digitale Transformation in Organisationen verantworten kann. Aufgabenbereiche und Anforderungen an den CDO wurden auf Basis von Analysen von Stelleninseraten im deutschsprachigen Raum sowie Experteninterviews in Deutschland und Österreich identifiziert. Die Ergebnisse zeigen, dass der Vollzug des digitalen Wandels im Unternehmen zu den zentralen Aufgaben des CDO zählt. Dazu ist es notwendig, digitale Trends zu beobachten, eine Digitalstrategie zu erarbeiten, effiziente Strukturen zu schaffen, persönliche Netzwerke zu pflegen sowie als Schnittstelle und digitaler Experte bereichsübergreifend zu agieren. Die Ergebnisse zeigen weiter, dass für die Wahrnehmung der Aufgaben annähernd dieselben Anforderungen gestellt werden wie jene, die auch für den CIO relevant sind (z. B. Technikkompetenz, betriebswirtschaftliche Kenntnisse, Projektmanagementkompetenz, Führungskompetenz und Soft Skills). Ein wesentlicher Befund ist zudem, dass die Erfahrung als aktiver Change-Manager wesentlich ist, um digitale Transformation erfolgreich steuern zu können. Schließlich legen die Untersuchungsergebnisse nahe, dass die Position des CDO idealerweise im Top-

Zusatzmaterial online Zusätzliche Informationen sind in der Online-Version dieses Artikels (doi: 10.1365/s40702-017-0320-7) enthalten.

M. Walchshofer · R. Riedl

Digital Business Management, Fakultät für Management, Fachhochschule Oberösterreich,

Wehrgrabengasse 1-3, 4400 Steyr, Österreich

R. Riedl $(\bowtie)$

Institut für Wirtschaftsinformatik - Information Engineering, Johannes Kepler Universität Linz,

Altenbergerstrasse 69, 4040 Linz, Österreich

E-Mail: rene.riedl@fh-steyr.at 
Management, jedenfalls aber in direkter Berichtslinie zum Chief Executive Officer (CEO) angesiedelt sein sollte, um volle Handlungseffektivität entfalten zu können.

Schlüsselwörter Aufgaben · Anforderungen · Chief Digital Officer · CDO · Chief Information Officer $\cdot \mathrm{CIO} \cdot$ Digitalisierung $\cdot$ Transformation

\section{The Chief Digital Officer: An Empirical Study}

Abstract The Chief Digital Officer, as a new management position, is increasingly established to plan and execute digital transformation. However, this development begs the question whether the Chief Information Officer (CIO) could also be responsible for this digital transformation in organizations. Responsibilities and requirements of the $\mathrm{CDO}$ have been identified on the basis of an analysis of job advertisements in German-speaking regions. Additionally, expert interviews with CDOs in Germany and Austria contributed to deeper insights. Results indicate that the CDO focuses on the execution of the digital transformation of a company. Therefore, the CDO is responsible for the definition of the corporate digital strategy, the monitoring of disruptive trends, the establishment of effective organisational structures, the management of the personal network, and the CDO furthermore serves as a cross-functional interface. Moreover, the results show that the requirements a CDO has to meet resemble those of the Chief Information Officer (CIO) (e. g., technical expertise, management know-how, project management expertise, leadership skills, and soft skills). A further finding is that experience in change management is a critical CDO skill to successfully manage digital transformation. Ideally, the CDO is positioned at the c-level, or at least directly reports to the Chief Executive Officer (CEO).

Keywords Chief Digital Officer · CDO - Chief Information Officer · CIO • Digitalization $\cdot$ Requirements $\cdot$ Responsibilities $\cdot$ Transformation

\section{Der CDO als neuartige Management-Position}

Die digitale Transformation verändert gesellschaftliche und wirtschaftliche Bereiche. Für Unternehmen kann dieser Wandel die fundamentale Neuausrichtung des Geschäftsmodelles und die Reorganisation etablierter Geschäftsprozesse mittels disruptiver Technologien (z. B. Augmented Reality) bedeuten. Für Unternehmen ist es daher wichtig, Trends der Digitalisierung wie z. B. Big Data aktiv zu begegnen. Eine Möglichkeit, die digitale Transformation erfolgreich voranzutreiben, besteht in der Schaffung der Management-Position des Chief Digital Officer (CDO) (Grossman und Rich 2012). Weltweit schaffen Unternehmen diese Position, letztlich mit dem Ziel, dass der CDO den digitalen Wandel orchestrieren soll. International wurden in den letzten Jahren in diversen Unternehmen CDOs bestellt. Mittlerweile bildeten sich sogar Interessensvereinigungen wie der CDO-Club, der 2011 gegründet wurde und aktuelle Informationen zu dieser neuartigen Position bereitstellt; der Fokus liegt 
hierbei auf dem US-amerikanischen Raum, vgl. z. B. die CDO-Talent-Map 2013 (CDO-Club 2014).

Der neuen Position des CDO steht der Wandel der Position des Chief Information Officer (CIO) gegenüber. Die digitale Transformation hat auch zu Veränderungen in den Aufgabenbereichen und Verantwortlichkeiten des CIO geführt. Die Spanne dieser Rolle reicht aktuell vom C-Level-Manager, der auf Unternehmensstrategie, Geschäftsprozesse und den digitalen Wandel fokussiert ist, bis hin zum Technikverantwortlichen, der auf Kostenminimierung achtet und ausschließlich den Betrieb der IT-Infrastruktur verantwortet (Weill und Woerner 2013; Chun und Mooney 2009).

Zunehmend stellt man sich in der Praxis die Frage, ob ein CDO tatsächlich notwendig ist, um die digitale Transformation in Unternehmen zu steuern, oder ob nicht auch der CIO dafür verantwortlich sein sollte. Im Diskurs zur Beantwortung dieser Frage gehen die Meinungen weit auseinander. Samuels (2015) hat dazu mehrere Stellungnahmen von Marktforschern und Praktikern untersucht und kommt zu dem Schluss, dass IDC und Gartner eine stark ansteigende Bedeutung des CDO prognostizieren, wohingegen Forrester sowie viele CIOs die Bedeutung des CDO in Frage stellen. Eine eher kritische Sichtweise zur Bedeutung und Notwendigkeit des CDO kann auch im akademischen Diskurs festgestellt werden. Gerth und Peppard (2016, S. 61) schreiben beispielsweise: „Some commentators have even suggested that it is now time to replace the CIO role with that of CDO (Chief Digital Officer). This line of thinking ignores the inherent organizational dynamics that lead to the derailment of the executive in charge of IT; merely changing the job title won't fix the problem." Angeheizt wird die Diskussion zudem durch Beiträge, die CIOs davor warnen, sich nicht vom CDO den Rang ablaufen zu lassen. Woods (2014, S. 1) spricht beispielsweise folgende Warnung aus: „Attention CIOs ... the best part of your job is under attack. If you don't get ahead of the trend toward the Chief Digital Officer role, your job may become a technology backwater, which is never what you had in mind."

Vor dem Hintergrund des aktuellen Diskurses zum CDO und seinem Verhältnis zum CIO wird im vorliegenden Beitrag die neuartige Management-Position des CDO näher untersucht. Vor allem werden die Aufgaben und Anforderungen beschrieben und die mögliche Einordnung in die Unternehmensorganisation wird diskutiert; weiter werden die Unterschiede und Gemeinsamkeiten von CDO und CIO erläutert. Die Datengrundlage des vorliegenden Beitrags bilden eine Sichtung der einschlägigen Fachliteratur, eine qualitative Analyse von CDO-Stelleninseraten im deutschsprachigen Raum sowie Ergebnisse von Interviews mit CDOs in Deutschland und Österreich.

\section{Stand der CDO-Forschung}

Die CDO-Talent-Map 2013 zeigt erstmalig in umfassender Weise Zahlen und Fakten zur Position des CDO. Demnach gab es im Jahr 2013 bereits etwa 500 Personen weltweit, die unter dem Jobtitel CDO agierten. Von diesen 500 Personen war die Mehrheit in Nordamerika tätig. Im darauffolgenden Jahr änderte sich dieses Verhältnis. In Europa stieg die Anzahl an CDOs seit 2013 signifikant an. Die Branchen- 
verteilung der CDOs weltweit zeigt ein klares Bild. In der Werbebranche werden die meisten CDOs angestellt, gefolgt von den Branchen Medien und Verlagswesen. Doch auch andere Sektoren wie Non-Profit, Einzelhandel sowie Finanzdienstleistungen holen sich immer öfter einen CDO ins Management, um den digitalen Wandel zu bewerkstelligen. Der CDO-Talent-Map 2013 zufolge ist der typische CDO männlich und zwischen 40 und 49 Jahre alt. CDOs haben unterschiedliche Ausbildungen und Berufserfahrung. Die meisten jedoch haben Expertise im Bereich Marketing, Vertrieb/Logistik, Technologie oder Consulting (CDO-Club 2014).

Neben der bloßen Darstellung deskriptiver Faktoren wie Verbreitung von CDOs und ihre Verteilung nach Branchen werden zunehmend öfter auch erklärende sowie normative Arbeiten veröffentlicht. In einer Studie des Beratungsunternehmens PWC widmeten sich Friedrich et al. (2015) unter anderem dem Zeitpunkt für den Einsatz eines CDO im Unternehmen. Es wird argumentiert, dass dieser Zeitpunkt primär vom jeweiligen Niveau der Digitalisierung eines Unternehmens abhängt. Befindet sich ein Unternehmen erst in der Entdeckungsphase (discovery phase) der Digitalisierung und begegnet es dieser Phase durch den Einsatz erster digitaler Applikationen ohne Koordination und ohne Kontrolle, so ist die Notwendigkeit der Schaffung der Position eines CDO nicht akut gegeben. Ebenso wenig ist Bedarf für die Schaffung, wenn ein Unternehmen die digitale Transformation (transformation phase) bereits komplett vollzogen hat und somit die Digitalisierung das Unternehmen durchdrungen hat und die gesamte Wertschöpfung digital gestaltet ist. Anders ist die Situation jedoch in den Phasen des Aufbaus (construction phase) und der Industrialisierung (industrialization phase). In der Aufbauphase kann ein CDO für die Schaffung einer Vision zur digitalen Transformation verantwortlich sein, Initiativen starten, für adäquate Strukturen und Programme sorgen und dazugehörige Standards setzen. In der darauffolgenden Industrialisierungsphase kann der CDO maßgeblich zur Veränderung der IT-Funktionen beitragen, Messgrößen zur Datenanalyse definieren und eine tiefere Digitalisierung auch in anderen Funktionen und Anwendungsbereichen im Unternehmen forcieren (Friedrich et al. 2015).

Was in Studien von Beratungs- und Marktforschungsunternehmen oft unbeachtet bleibt, ist die Frage, ob die Rolle des CDO tatsächlich notwendig ist oder ob es nicht in der Verantwortung des CIO liegt, die digitale Transformation in Unternehmen zu steuern. Die akademische Forschung befasst sich hier im Gegensatz zu Studien aus der Praxis mit der Thematik in einer tiefergehenden Weise. Beispielsweise argumentieren Gerth und Peppard (2016, S. 62) auf der Basis von Befunden empirischer Forschung wie folgt: „Indeed, frustrated by the perceived inability of their CIOs to drive the digital agenda, some organizations are now either replacing them with Chief Digital Officers (CDOs) or hiring CDOs specifically to drive their digital initiatives ... This practice only serves to demonstrate the naïveté within many management teams, which operate under the misguided belief that the digital realm is different than good old-fashioned IT. The irony is, when one reads the job specification for this role, it clearly mirrors what a CIO should be doing." Die von Gerth und Peppard angesprochene ,,job specification“"wird im Folgenden näher betrachtet, gruppiert in Aufgaben und Anforderungen. 


\subsection{Aufgaben}

CDOs tragen nach Friedrich et al. (2015) Verantwortung für die gesamte digitale Transformation eines Unternehmens. Somit zeichnet der CDO für die technische Infrastruktur und das Datenmanagement, den kulturellen Organisationswandel sowie die digitale Organisation und Steuerung, welche auf das operative Geschäft, den Kunden und die Produkte abgestimmt sein soll, verantwortlich. Konkret entstehen Aufgaben durch die Einführung von digitalen Innovationen zur Interaktion mit Kunden, Lieferanten und Partnern und zur Wandlung der Unternehmenskultur und -organisation.

Horlacher und Hess (2016) beschreiben die Aufgaben des CDO als sehr nachfrageorientiert. So soll die Person strategische Optionen zum Unternehmens-, Marktund Erlöswachstum aufzeigen und das in erster Linie zur Schaffung IT-basierter Innovationen. Neben der Entwicklung einer Digitalstrategie soll der CDO den notwendigen Wandel im Unternehmen koordinieren und dabei die gesamte Belegschaft motivieren und entsprechend trainieren und befähigen. Im Rahmen der Digitalisierung sollen vom CDO ausgehend eingefahrene Mentalitäten und ein traditionelles Silo-Denken aufgebrochen werden. Der CDO sollte auch interne und externe Netzwerke und Beziehungen aufbauen, erweitern und pflegen.

Naimi (2012) beschreibt wie Horlacher und Hess die Verantwortlichkeiten des CDO als sehr nachfrageorientiert. Der CDO ist für die gesamte digitale Kundenerfahrung verantwortlich. Die Gestaltung einer personalisierten und interaktiven Kundenansprache über alle Kanäle hinweg (digital customer journey) gehört zu seinen wesentlichen Aufgaben.

Um einen Wandel im Unternehmen zu vollziehen und eine entsprechende Aufbruchsstimmung im Unternehmen zu erzeugen, liegt es nach Ansicht einer Veröffentlichung von McKinsey (Rickards et al. 2015) in der Verantwortung des CDO, Agilität und Tempo zu fordern und zu fördern. Ein höheres Prozesstempo kann durch kürzere Zielerreichungszeiten realisiert werden. Weiter können durch flexible Budgetverwaltung und rasche Ressourcenzuteilung Projekte bzw. Produkte, die erste Erfolgsanzeichen zeigen, schnell skaliert werden.

\subsection{Anforderungen}

Dumeresque (2014) beschreibt die Eigenschaften und Fähigkeiten des CDO mit einer kundenzentrierten Denkweise und sehr guten Kenntnissen der Technologielandschaft. Zudem sollte der CDO bedeutende Berufs- und Management-Erfahrung aufweisen können und Wissen und Erfahrung im Change-Management besitzen.

Horlacher und Hess (2016) nennen neben IT-Know-how starke Fähigkeiten in Strategieentwicklung, Change-Management und Kommunikation als wesentliche Anforderungen. Außerdem soll der CDO kundenzentriertes Verständnis für kaufmännische und betriebswirtschaftliche Aspekte haben (z. B. Geschäftsmodelle, Prozesse und Kundenbedürfnisse). Als guter Kommunikator sollte der CDO fähig sein, die strategische Vision sowie Ziele gegenüber Mitarbeitern und Kollegen zu artikulieren. Überdies sollte ein CDO die Fähigkeit besitzen, über Abteilungsgrenzen hinauszudenken, das Unternehmen als Ganzes zu sehen und funktionsübergreifend 
zu handeln. Durch das Überwinden vom Denken in abgegrenzten Silos können unterschiedliche Abteilungen verbunden und Synergien entwickelt werden.

Grossmann und Rich (2012) präzisieren die nötige digitale Expertise eines CDO. Erfahrung in E-Commerce, Online-Marketing und Social Media sind unumgänglich. Als empathische Führungspersönlichkeit sollte der CDO zudem über internationale Erfahrung verfügen.

\subsection{Fazit zur bisherigen CDO-Forschung}

Eine Sichtung der aktuell vorliegenden Publikationen zum CDO zeigt, dass ein Großteil der Studien von Beratungs- und Marktforschungsunternehmen veröffentlicht wurde. Weiter existieren Studien von Interessensvereinigungen, allen voran sind hier die vorwiegend deskriptiven Untersuchungen des CDO Club zu nennen. Forschungsbefunde, die auf der Basis anerkannter empirischer Forschungsmethoden erarbeitet wurden (vgl. z. B. Horlacher und Hess 2016) und die somit als wissenschaftlich zu charakterisieren sind, gibt es bislang kaum. Inhaltlich fällt auf, dass die von Beratungs- und Marktforschungsunternehmen sowie von Interessensvereinigungen publizierten Studien in der Regel nicht hinterfragen, ob die Rolle des CDO überhaupt notwendig ist. Das Infragestellen der Rolle des CDO ergibt sich aus dem Umstand, dass nach Ansicht mancher Autoren (z. B. Gerth und Peppard 2016) existierende CIO-Rollenbeschreibungen bereits die wesentlichen Aufgaben eines CDO umfassen. Verschärft wird der aktuelle Diskurs durch den Umstand, dass das Thema „Digitalisierung“ Eigenschaften eines Modethemas hat (vgl. Steininger et al. 2009). Mertens und Barbian (2015) kritisieren mit Blick auf das Thema „Digitalisierung“ unter anderem ,die Subsumption von Vorhandenem“. Es wird also etwas bereits Vorhandenes in eine neue Mode übernommen. Umgelegt auf den Kontext des vorliegenden Beitrags stellt sich somit die Frage, ob die Management-Position des CDO tatsächlich neu ist oder lediglich neuartig erscheint, weil bereits Vorhandenes (die Rollenbeschreibung des CIO inklusive seiner Aufgaben und Anforderungen) unter einem neuen Begriff (Chief Digital Officer bzw. CDO) diskutiert wird. Im Folgenden wird über eine empirische Untersuchung berichtet, die einen Beitrag zur Beantwortung dieser Frage leistet.

\section{Methodik der empirischen Untersuchung}

Um tiefere Einblicke in die CDO-Rolle sowie Aufgaben und Anforderungen zu erlangen, wurden (i) Stelleninserate in Deutschland, Österreich und der Schweiz analysiert und (ii) Experteninterviews mit CDOs in Deutschland und Österreich geführt.

Bei der Analyse des Stellenmarktes nach offenen CDO-Positionen lag der Fokus auf Online-Jobbörsen sowie Suchmaschinen und Karriereseiten von Tageszeitungen im DACH-Raum. Dabei wurde auf die Bekanntheit und Marktpräsenz der Jobportale und Tageszeitungen geachtet. Zur 3-monatigen Überwachung (01.02.2016-30.04.2016) der Stelleninserate wurden Job-Alerts mit der Wortkombination „Chief Digital Officer“ bei folgenden Online-Jobportalen und Tageszeitungen 
angelegt: monster.de, jobisjob.de, monster.at, karriere.at, monster.ch und jobs.ch sowie Frankfurter Allgemeine Zeitung, Süddeutsche Zeitung, Der Standard, Die Presse, Neue Zürcher Zeitung und Tages-Anzeiger. Durch Archivanfragen und Kontaktierung eines führenden Anzeigendienstleisters konnte der Beobachtungszeitraum der Stelleninserate auf 40 Monate (01.01.2013-30.04.2016) ausgeweitet werden. Insgesamt konnten so 18 Stellenausschreibungen identifiziert werden, von denen fünf nicht weiter analysiert wurden, da der Jobtitel den Begriff „Chief Digital Officer“ zwar beinhaltete, jedoch Zusätze wie „Referent des“, ,Personal Assistant“ oder „Executive Assistant to the“ vorangestellt waren. Die 13 im Detail untersuchten Stellenausschreibungen bezogen sich auf folgende Branchen und Staaten: Dienstleistungsbranche (6), Industrie (2), Medien (2), Konsumgüter (2) und Politik (1) sowie Deutschland (10), Österreich (2) und Schweiz (1).

Zur Kontaktaufnahme mit möglichen Interviewpartnern wurden Kontaktdaten von CDOs im deutschsprachigen Raum gesammelt. Die sozialen Geschäftsnetzwerke Xing und LinkedIn, Fachartikel in Online-Medien (z. B. cio.de) und UnternehmensWebsites waren Quellen der Suche. Es konnten so 52 CDOs (Stand: März 2016) in Deutschland, Österreich und der Schweiz aus unterschiedlichen Branchen identifiziert werden. Davon wurden 18 CDOs für ein Experteninterview kontaktiert, sodass sich die Branchenverteilung aus der Grundgesamtheit widerspiegelt. Sechs Interviews mit CDOs aus Deutschland und Österreich konnten letztendlich in den Monaten März und April 2016 geführt werden. Konkret wurden 4 CDOs in Deutschland aus den Branchen Dienstleistung (2), Medien (1) sowie Marketing und Werbung (1) persönlich befragt. In Österreich stand jeweils ein CDO aus der Bankenund Versicherungsbranche sowie aus Marketing und Werbung zur Verfügung. Im Mittelpunkt der Befragung standen die Klärung der Aufgaben und Anforderungen sowie die organisatorische Einordnung der Position in der Unternehmenshierarchie. Die Interviews dauerten im Durchschnitt 30 min, wurden digital aufgezeichnet und anschließend transkribiert. Diese Transkripte dienten wiederum als Grundlage zur weiteren qualitativen Analyse der Interviews. Zur einheitlichen Analyse wurde jeweils ein Kodierleitfaden mit Kategoriensystem für Aufgaben und Anforderungen erstellt. Anhand der beiden Leitfäden wurden Textelemente aus Stelleninseraten den definierten Kategorien zugeordnet.

Schlussendlich wurden die Daten aus der Analyse der Stelleninserate und der transkribierten Interviews zu einem Rollenprofil des CDO zusammengeführt. Auf Basis dieses Rollenprofiles kann der CDO dem CIO gegenübergestellt werden. Alle Stelleninserate sowie der Interviewleitfaden stehen als Online-Material zur Verfügung.

\section{Ergebnisse der Untersuchung}

\subsection{Aufgaben}

Der CDO ist für die Erarbeitung der Digitalstrategie verantwortlich. Diese Strategie wird von der Unternehmensstrategie abgeleitet und soll vom CDO umgesetzt und im Unternehmen verankert werden. Der CDO ist zudem für die digitale Transforma- 
tion, also für die Steuerung des digitalen Wandels im Unternehmen verantwortlich (vgl. Matt et al. 2015). Eine wesentliche Aufgabe des CDO ist weiter die Prüfung und Erarbeitung alternativer, disruptiver Geschäftsmodelle im Digitalbereich. Durch Akquisitionen von Start-ups, strategische Partnerschaften oder neue disruptive Innovationen sollen neue Geschäftsmodelle entstehen und zum Wachstum bzw. zur Geschäftsentwicklung beitragen.

Zentrale Aufgabe des CDO ist auch die Schaffung effizienter Strukturen. Durch Digitalisierung und Vereinfachung von Geschäftsprozessen soll ein höheres Prozesstempo erreicht und Agilität sichergestellt werden. Effizientere Strukturen betreffen sowohl interne Arbeitsabläufe als auch die Interaktion mit Externen wie z. B. Kunden und Lieferanten. Durch die Nachfrageorientierung des CDO (vgl. Horlacher und Hess 2016) liegt ein Fokus auf der Gestaltung der Customer Journey.

Die Änderung der Unternehmenskultur soll der CDO aktiv mitgestalten. Durch Unterstützung und Leitung eines Change-Prozesses soll ein Mentalitätswandel und kultureller Wandel im Unternehmen bereichsübergreifend vollzogen werden. Neben der Mitarbeiterführung und-motivation ist auch die Mitarbeiterentwicklung Aufgabe des CDO. Der CDO soll Mitarbeiter hinsichtlich Digitalisierungsagenden befähigen, trainieren und deren Kompetenzen erweitern.

Der CDO soll interne und externe Netzwerke pflegen und ausbauen. Das ist auch in Anbetracht seiner Aufgabe als Schnittstellenkoordinator wichtig. Enge Abstimmungen mit der Geschäftsführung, dem CIO und den Fachbereichen liegen im Aufgabenbereich des CDO. Eine weitere Aufgabe liegt in der Repräsentation nach außen.

Der CDO tritt als Leiter von Projekten im digitalen Bereich auf. Die Projektentwicklung, -umsetzung und -leitung unterstreicht seine Schnittstellenfunktion bei bereichsübergreifenden Projekten. Zur Arbeit des CDO gehört explizit auch die Überprüfung des Fortschrittes von Projekten. Durch Controlling mittels geeigneter Kennzahlen soll der Erfolg der Umsetzung der Digitalstrategie geprüft werden. Der CDO soll Technologietrends beobachten und deren mögliches Potenzial für das Unternehmen erkennen. Hier schließt sich ein Kreis und führt wieder zur Prüfung und Erarbeitung alternativer, disruptiver Geschäftsmodelle, die durch neue Technologien möglich werden.

\subsection{Anforderungen}

In allen Stelleninseraten wird ein abgeschlossenes wirtschaftliches oder technisches Studium oder mehrjährige Berufserfahrung in einem ähnlichen Umfeld gefordert. Dieses Bild spiegelt sich in den Ausbildungen und Erfahrungen der Interviewpartner wider. Alle Interviewpartner haben ein Hochschulstudium absolviert oder eine Ausbildung im geforderten Umfeld abgeschlossen. Abhängig von der Branche und der Unternehmensorganisation ist ein technisches Grundverständnis bis hin zu einer tiefen Technik- und IKT-Kompetenz eine wesentliche Jobanforderung. Diese Kompetenz sollte auf einem Hochschulstudium bzw. auf Berufserfahrung beruhen. Ein abgeschlossenes Studium im Wirtschaftsbereich (z. B. Betriebswirtschaft) und/oder dementsprechende Berufserfahrung prägen das geforderte geschäftsorientierte Denken. Zudem ist Wissen in Teilbereichen der Betriebswirtschaft wie z. B. im Personal- 
management, Geschäftsprozessmanagement sowie Marketing und Vertrieb ebenso wichtig wie Verständnis für Geschäftsstrategien. Ein digitales Know-how ist unumgänglich und breitgefächert. Wiederum abhängig von der Branche soll der CDO Experte im digitalen Fachbereich sein und über aktuelles Wissen zu technologischen Trends verfügen. Das digitale Know-how kann durch Berufserfahrung im Digitalbereich (z. B. in einem Start-up) erworben worden sein. Idealerweise hat die Person Erfahrung in der Umsetzung von Digitalisierungsstrategien. Als Leiter und Unterstützer bei Change-Prozessen soll die Person Erfahrung im Change-Management aufweisen. Dazu sind Einfühlungsvermögen ergänzt um Verhandlungsgeschick und mediatorische Fähigkeiten genauso nötig wie Durchsetzungskraft. Eine gewisse Frustrationstoleranz, der Wille bei Rückschlägen weiterzumachen und hohe Eigenmotivation sind laut Angaben der Interviewpartner in Änderungsprozessen als individuelle Fähigkeiten nötig. Die Fähigkeit, funktionsübergreifend zu denken, spiegelt sich in gedanklicher Flexibilität und der gedanklichen Überwindung von Abteilungsgrenzen im Unternehmen. Offenheit und Agilität sind dazu ebenso hilfreich wie Innovationskraft und „Out-of-the-box“-Denken. Zur Erfüllung seiner Aufgabe als Schnittstellenkoordinator soll die Person über starke Kommunikationsfähigkeiten verfügen. Schließlich zeigt die Analyse der Daten, dass Führungserfahrung sowie die Fähigkeit, sich selbst und andere zu motivieren, vorausgesetzt werden.

\subsection{Einordnung in die Unternehmensorganisation}

In der Fachliteratur wird eine Einordnung des CDO in direkter Berichtslinie zum CEO vorgeschlagen, sofern nicht überhaupt eine Verankerung auf Vorstandsebene gefordert wird (Dumeresque 2014; Zisler et al. 2016). In acht Stelleninseraten wird diese Positionierung in direkter Berichtslinie auch angegeben. Unterstrichen wird diese Positionierung von den Interviewpartnern. Alle sechs Personen gaben an, hierarchisch dem CEO direkt untergeordnet zu sein. Alle Interviewpartner untermauerten zudem, dass digitale Agenden auf Top-Management-Ebene oder direkt darunter angesiedelt sein sollten, um strategischen Einfluss auf oberster Ebene wirksam ausüben zu können.

\section{Unterschiede und Gemeinsamkeiten von CDO und CIO}

Im zweiten Abschnitt dieses Beitrags wurde die Frage gestellt, ob die CDO-Rolle tatsächlich neu ist oder nur neuartig erscheint, weil lediglich - so die Ansicht mancher Autoren (z. B. Gerth und Peppard 2016) - CIO-typische Aufgaben und Anforderungen unter dem Begriff „Chief Digital Officer“ bzw. „CDO“ diskutiert werden. Handelt es sich also bei der CDO-Position lediglich um, ,alten Wein in neuen Schläuchen"? Nachfolgend wird auf der Basis der Ergebnisse der vorliegenden Studie und unter Bezugnahme auf CIO-Rollenbeschreibungen (vgl. z. B. Heinrich et al. 2014, S. 73-84; Krcmar 2015, S. 466-472) diskutiert, welche Unterschiede und Gemeinsamkeiten von CDO und CIO aktuell bestehen.

Ähnlichkeit in den Aufgaben herrscht bei zukunftsweisenden Überlegungen und Ausführungen in Form der Visions- bzw. Strategieentwicklung; ebenso sollen bei- 
de Rollen neue Trends und Technologien und deren Potenziale erkennen. Hier ist das Aufgabenspektrum des CDO aber etwas breiter gestaltet. Der CDO soll neue Trends und Technologien nicht nur erkennen, sondern grundlegende Neuerungen und Innovationen schaffen und disruptive Geschäftsmodelle entwickeln. Bei der Aufgabe der Effizienzsteigerung liegt der Tätigkeitsschwerpunkt des CIO auf der Verbesserung der Leistung der technischen Informationsinfrastruktur (z. B. weniger Systemausfälle und kürzere Systemantwortzeiten) und der Verbesserung von Geschäftsprozessen durch die IT (Sackarendt 2003). Der CDO fokussiert hingegen auf interne und externe Verbesserungen. Durch seine Kundenzentrierung sollen nicht nur interne Strukturen und Abläufe verbessert werden, sondern auch externe wie z. B. die Vereinfachung der Customer Journey. Weiter stehen beim CIO bei der Mitarbeiterführung und -entwicklung die IT-Abteilung und deren Belegschaft im Mittelpunkt (Thiemann 2014). Der Tätigkeitsbereich des CDO ist hingegen nicht auf einzelne Abteilungen beschränkt, sondern betrifft alle Bereiche eines Unternehmens. Digitalagenden sollen global im Unternehmen verankert werden. Dieser übergeordnete Tätigkeitsbereich spiegelt sich in der Aufgabe der Schnittstellenkoordination wider. So liegt es im Aufgabenbereich des CDO, interne und externe Schnittstellen zu harmonisieren. Ähnliche Aufgaben erledigen CIO und CDO im IT-Controlling sowie im IT-Projektmanagement. Die weiteren Aufgaben des CIO betreffen allesamt die Sicherung und Vernetzung der IT sowie die Gestaltung und Entwicklung von Geschäftsprozessen und Anwendungen (Penzel 2001). Dem gegenüber stehen die weiteren Aufgaben des CDO. Diese betreffen kommunikative Aspekte, wie Repräsentation nach außen oder aktives Netzwerken und strategische Aspekte, wie Wandlung der Unternehmenskultur und gesamte Steuerung der Digitalisierung.

Hinsichtlich der Anforderungen wurde festgestellt, dass CDO und CIO jeweils über Technikkompetenz, betriebswirtschaftliche Kenntnisse in Kombination mit geschäftsorientiertem und strategischem Handeln und Denken, aber auch über Führungskompetenz verfügen sollten (Portela et al. 2010). Zudem ist sowohl für den CDO als auch den CIO Projektmanagementkompetenz unerlässlich. Die Soft Skills sind beinahe gänzlich übereinstimmend. Eine Abgrenzung des CDO zum CIO kann im Aktivitätsniveau während eines Change-Prozesses vorgenommen werden. So ist der CDO aktiver Change-Manager. Bei der Aufgabe „Wandlung der Unternehmenskultur" leitet und steuert der CDO den Prozess aktiv und dafür ist Erfahrung in Change-Management-Prozessen erforderlich. Eine solch aktive Rolle bei Veränderungsprozessen wird in der Regel vom CIO nicht erwartet (auch wenn in manchen Quellen die Rolle des CIO als Change-Manager explizit genannt wird, z. B. in Krcmar 2015, S. 468-469).

Die wesentlichen Unterschiede und Gemeinsamkeiten von CDO und CIO hinsichtlich Aufgaben und Anforderungen sind in Abb. 1 im Überblick dargestellt. Man sieht, dass es sowohl bei den Aufgaben als auch bei den Anforderungen signifikante Schnittmengen gibt. Es wird jedoch auch transparent, dass CDO- und CIOspezifische Aufgaben existieren. Es fällt zudem auf, dass es keine Anforderungen an den CIO gibt, die nicht auch für den CDO relevant wären. Insgesamt kann bei den Anforderungen festgestellt werden, dass kaum Unterschiede existieren (Ausnahme: Aktivität bei Veränderungsprozessen). Zu beachten ist, dass es sich bei den dargestellten CDO-Aufgaben und -Anforderungen um die Summe der durch die Analyse 


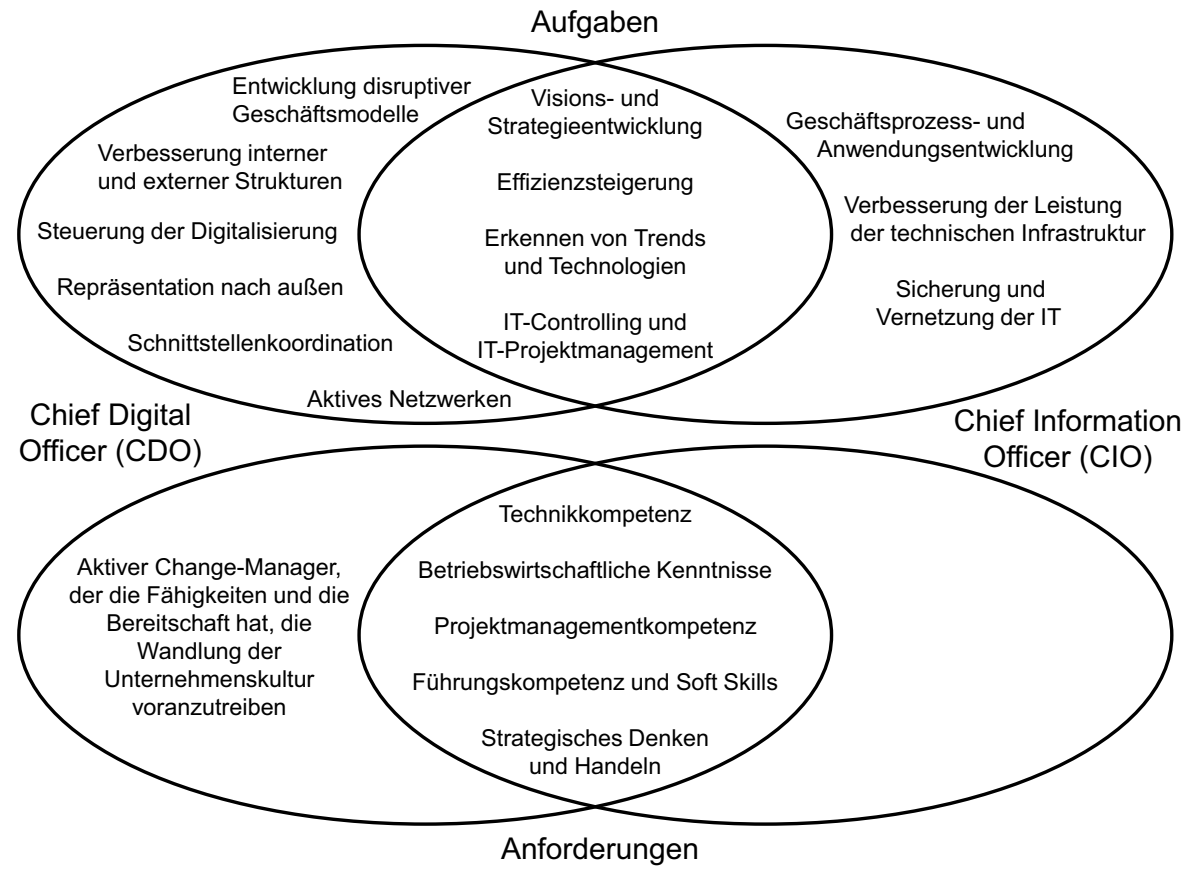

Abb. 1 Unterschiede und Gemeinsamkeiten von CDO und CIO

der Stelleninserate und Interviews gewonnenen Erkenntnisse handelt, so dass im Einzelfall in einer spezifischen Organisation in der Regel nicht alle genannten Aufgaben und Anforderungen zum Tragen kommen werden.

Als Resümee kann die Arbeit des CDO im Vergleich zu jener des CIO als übergeordnet (im Sinne von strategischer ausgerichtet) bezeichnet werden. Die Person ist funktionsübergreifend tätig und die Digitalisierung bzw. die digitale Transformation des Unternehmens stehen im Fokus. Das unterstreicht die Erkenntnis von Horlacher und Hess (2016), wonach der CDO für strategische Aspekte verantwortlich ist, während der CIO oft für organisatorische und technische Aspekte verantwortlich ist. Untersuchungen zeigen zudem, dass im deutschen Sprachraum der CIO in vielen Fällen nicht Mitglied der Geschäftsleitung ist (Riedl et al. 2008; Hütter und Riedl 2011). Dies ist auch ein Indikator dafür, dass viele CIOs nicht (primär) auf der strategischen Ebene agieren.

Ergebnisse aktueller Forschung legen jedoch nahe, den Befund, dass der CDO dem CIO als übergeordnet anzusehen ist, kritisch zu hinterfragen. Eine aktuelle Synopse, in der 98 Fachartikel zur CIO-Thematik aus den letzten drei Jahrzehnten analysiert wurden, zeigt, dass CIOs in unterschiedlichen Rollen agieren (Hütter und Riedl 2017). Manche dieser Rollen sind eher operativ-administrativ angelegt (,Technology Provider" oder „Integration Advisor"), andere eher strategisch („Business Thinker“ oder „Innovation Driver“). Weiter zeigt diese Synopse, dass von CIOs zunehmend erwartet wird, in allen, zumindest aber in mehreren Rollen zu agieren. Zudem legen die Ergebnisse von Hütter und Riedl (2017) nahe, dass eine Liste aller in der Fachli- 
teratur bislang formulierten CIO-Aufgaben de facto auch alle Erwartungen abdeckt, die aktuell an CDOs gestellt werden.

$\mathrm{Ob}$ es sich also bei der CDO-Position um ,alten Wein in neuen Schläuchen“ handelt, hängt entscheidend davon ab, ob als Vergleichsmaßstab Einzelarbeiten zu CIOAufgaben herangezogen werden oder eine Liste aller in der gesamten Fachliteratur genannten CIO-Aufgaben und -Anforderungen. Tut man letzteres, dann würden die in Abb. 1 links dargestellten CDO-spezifischen Aufgaben und Anforderungen leere Mengen darstellen, weil sich die dort genannten Aufgaben und Anforderungen in die mittig dargestellten Schnittmengen verschieben würden. In diesem Zusammenhang sei auch erwähnt, dass aktuelle Beiträge zur CIO-Thematik in hoch gerankten Fachzeitschriften Aussagen enthalten, die nahe legen, dass heutzutage eher dem CDO zugeschriebene Aufgaben mittel- bis langfristig in das Aufgabenportfolio des CIO übergehen werden. Benlian und Haffke (2016) schreiben: „Mid to long term, CIOs might be able to reduce or fully remove negative prejudices by demonstrating their high level of business understanding and proactively guiding business strategy, particularly by bringing in their perspective on emerging technology trends, IT-driven innovation, and digital transformation ... the CIO's responsibilities are expected to grow in an increasingly digital economy“ (S. 116f.).

\section{Der CDO - ein zeitlich begrenztes Phänomen?}

Die geänderten Verantwortungs- und Aufgabenbereiche des CIO in Zeiten der digitalen Transformation führen zu teilweisen Überschneidungen mit den Aufgaben des CDO. Eine Abgrenzung der beiden Positionen zeigt, dass der CDO eine übergeordnete Sicht auf unterschiedliche Agenden und Bereiche im Unternehmen hat. Als aktiver Gestalter und Treiber der Transformation und der Kulturänderung ist es eine seiner Aufgaben, Veränderungsprozesse aktiv zu steuern. Verstärkt wird diese Aufgabe durch die Schnittstellenkoordination des CDO. Im Gegensatz zum CIO zeichnet sich der CDO durch eine kundenzentrierte Denkweise aus und ist eher nachfrageorientiert als angebotsorientiert (Horlacher und Hess 2016). Die Anforderungen an die beiden Positionen sind nahezu identisch. Ein durch Hochschulstudium oder mehrjährige Berufserfahrung erlangtes Wissen in Technik und Betriebswirtschaft ergänzt um Führungskompetenz und Soft Skills zeichnen sowohl CDOs als auch CIOs aus.

Es bleibt abzuwarten, wie sich die Rolle des CDO weiterentwickeln wird. Dazu sollte diese Management-Position auch in den kommenden Jahren beobachtet und analysiert werden. Wenn in Zukunft mehr und mehr Unternehmen die vier Phasen der digitalen Revolution durchlaufen haben und somit die Transformation vollzogen ist und die Digitalisierung alle Bereiche eines Unternehmens durchdrungen hat (siehe Friedrich et al. 2015), dann sind im Wesentlichen jene Aufgaben des CDO obsolet, die ihn heute vom CIO unterscheiden. Es könnte daher sein, dass aus zukünftiger Sicht rückblickend gesehen die Position des CDO eine temporär begrenzte Erscheinung der digitalen Revolution war.

Eine wesentliche Implikation der vorliegenden Studie für die Praxis ist zudem, dass Unternehmen nicht vorschnell einen CDO installieren sollten. Wenn es im Unternehmen bereits einen CIO gibt, der die an einen CDO gestellten Anforderungen 
erfüllt, dann kann auch diese Person den digitalen Wandel orchestrieren. Hierbei sollte man sich jedoch folgende Fragen stellen:

- Ist der CIO vom Wesen her ein aktiver Change-Manager, der den Wandel effektiv und effizient umsetzen kann?

- Wenn nicht, kann sich der CIO durch Qualifizierungsmaßnahmen ausreichend kompetent machen, ist also ein Hineinwachsen in die CDO-Rolle möglich?

- Hat der CIO überhaupt Ambitionen, aus seiner oftmals eher passiven Rolle, die sich vorwiegend mit administrativen und teilweise operativen Aufgaben des ITManagements befasst, in eine eher aktive Rolle mit Fokus auf strategische Aufgaben und organisationale Transformation zu schlüpfen?

- Verfügt der CIO überhaupt noch über ausreichende zeitliche Ressourcen, um sein Aufgabenspektrum zu erweitern?

- Wenn nicht, können durch Umgestaltung der IT-Organisation im Allgemeinen sowie des Arbeitsumfeldes im Speziellen (z. B. durch Schaffung zusätzlicher ITProjektleiterstellen) ausreichend zeitliche Ressourcen geschaffen werden, damit der CIO zusätzliche Aufgaben der digitalen Transformation wahrnehmen kann?

Diese und weitere Fragen müssen die Verantwortlichen beantworten, um zu entscheiden, ob ein aktuell vorhandener CIO auch die mit der digitalen Transformation einhergehenden Aufgaben übernehmen sollte. Entscheidet man sich hingegen dafür, in der Organisation neben einem bereits vorhandenen CIO einen CDO zu installieren, so ist zu beachten, dass damit auch Konfliktpotenzial geschaffen wird. Weisungsbefugnisse, Zugriff auf Ressourcen personeller und finanzieller Art, Zielkonflikte sowie Absicherung und Ausbau von Macht sind hierbei nur einige Faktoren, die aufzeigen, dass es einer Reihe von Entscheidungen und Maßnahmen bedarf, wenn der CIO und CDO in einem Unternehmen wirksam zusammenarbeiten sollen. Wie auch immer Geschäftsleitungen in Zukunft den organisatorischen und personellen Rahmen für die digitale Transformation gestalten, fest steht jedenfalls, dass die voranschreitende Digitalisierung in vielen Unternehmen etablierte Organisationsformen verändern wird.

Open access funding provided by University of Applied Sciences Upper Austria.

Open Access Dieser Artikel wird unter der Creative Commons Namensnennung 4.0 International Lizenz (http://creativecommons.org/licenses/by/4.0/deed.de) veröffentlicht, welche die Nutzung, Vervielfältigung, Bearbeitung, Verbreitung und Wiedergabe in jeglichem Medium und Format erlaubt, sofern Sie den/die ursprünglichen Autor(en) und die Quelle ordnungsgemäß nennen, einen Link zur Creative Commons Lizenz beifügen und angeben, ob Änderungen vorgenommen wurden.

\section{Literatur}

Benlian A, Haffke I (2016) Does mutuality matter? Examining the bilateral nature and effects of CEOCIO mutual understanding. J Strateg Inf Syst 25:104-126

CDO-Club (2014) Chief digital officer talent map 2013. http://cdoclub.com. Zugegriffen: 29. Jan 2016

Chun M, Mooney J (2009) CIO roles and responsibilities: twenty-five years of evolution and change. Inf Manag. doi:10.1016/j.im.2009.05.005

Dumeresque D (2014) The chief digital officer: bringing a dynamic approach to digital business. Strateg Dir 30:1-3. doi:10.1108/SD-12-2013-0104 
Friedrich R, Péladeau P, Mueller K (2015) The 2015 chief digital officer study - adapt, disrupt, transform, disappear. In: PWC (Hrsg) Strategy\&. http://www.strategyand.pwc.com/reports/chief-digital-officerstudy. Zugegriffen: 29. Jan. 2016

Gerth AB, Peppard J (2016) The dynamics of CIO derailment: how CIOs come undone and how to avoid it. Bus Horiz 59:61-70

Grossman R, Rich J (2012) The rise of the chief digital officer. http://www.russellreynolds.com/insights/ thought-leadership/the-rise-of-the-chief-digital-officer. Zugegriffen: 20. Febr. 2016

Heinrich LJ, Riedl R, Stelzer D (2014) Informationsmanagement - Grundlagen, Aufgaben, Methoden. de Gruyter Oldenbourg, München

Horlacher A, Hess T (2016) What does a chief digital officer do? Managerial tasks and roles of a new C-level position in the context of digital transformation. Proceedings of the 49th Hawaii International Conference on System Sciences, HICSS, Hawaii, S 5126-5135 doi:10.1109/HICSS.2016.634

Hütter A, Riedl R (2011) Der Chief Information Officer (CIO) in Deutschland und den USA: Verbreitung und Unterschiede. Inf Manag Consult 26:61-66

Hütter A, Riedl R (2017) Chief Information Officer (CIO) role effectiveness: literature review and implications for research and practice. In: Becker J (Hrsg) SpringerBriefs in Information Systems. Springer International Publishing, Cham

Krcmar H (2015) Informationsmanagement, 6. Aufl. Springer Gabler, Berlin Heidelberg

Matt C, Hess T, Benlian A (2015) Digital transformation strategies. Bus Inf Syst Eng 57:339-343

Mertens P, Barbian D (2015) Digitalisierung und Industrie 4.0 - Moden, modische Überhöhung oder Trend? Arbeitsbericht 1/2015. Friedrich-Alexander-Universität, Erlangen-Nürnberg

Naimi O (2012) The emerging chief digital officer. Website Mag. http://www.oliviernaimi.com/theemerging-chief-digital-officer.html. Zugegriffen: 28. Januar 2016

Penzel H-G (2001) Hat der CIO im Vorstand eine Zukunft? Wirtschaftsinformatik 43:409

Portela L, Carvalho R, Varajão J, Magalhães L (2010) A review of chief information officer - main skills. In: Lytras MD, Ordonez de Pablos P, Ziderman A, Roulstone A, Maurer H, Imber JB (Hrsg.) Organizational, Business, and Technological Aspects of the Knowledge Society. WSKS 2010. Communications in Computer and Information Science 112. doi:10.1007/978-3-642-16324-1

Rickards T, Smaje K, Sohoni V (2015) Transformer in chief: the new chief digital officer. http://www.mckinsey.com/business-functions/organization/our-insights/transformer-in-chief-thenew-chief-digital-officer. Zugegriffen: 23. Mai 2016

Riedl R, Kobler M, Roithmayr F (2008) Zur personellen Verankerung der IT-Funktion im Vorstand börsennotierter Unternehmen: Ergebnisse einer inhaltsanalytischen Betrachtung. Wirtschaftsinformatik 50:111-128

Sackarendt M (2003) Der CIO aus dem Blickwinkel des Business. In: Gora W, Schulz-Wolfgramm C (Hrsg) Informationsmanagement - Handbuch für die Praxis. Springer, Berlin Heidelberg, S 157-170

Samuels M (2015) Chief data and digital officers rise to threaten the CIO. http://www.computerweekly.com/ feature/Chief-Data-and-Digital-Officers-rise-to-threaten-the-CIO. Zugegriffen: 23. Mai 2016

Steininger K, Riedl R, Roithmayr F, Mertens P (2009) Moden und Trends in Wirtschaftsinformatik und Information Systems: Eine vergleichende Literaturanalyse. Wirtschaftsinformatik 51:478-495

Thiemann S (2014) Der CIO im Wandel - vom Verwalter zum Gestalter. Heidrick \& Struggles (Hrsg). http://www.heidrick.com/ /media/Publications\%20and\%20Reports/Der-CIO-im-Wandel-Vom-Verwalter-zum-Gestalter.pdf. Zugegriffen: 23. Mai 2016

Weill P, Woerner S (2013) The future of the CIO in a digital economy. MIS Q Exec 12:65-75

Woods D (2014) Don't let a chief digital officer steal the best part of your job. Forbes. http://www.forbes.com/ sites/danwoods/2014/04/14/dont-let-a-chief-digital-officer-steal-the-best-part-of-your-job/. Zugegriffen: 24. Jan. 2017

Zisler K et al (2016) Chief digital officer. Z Führung Org 85:76-83 